CASO CLÍNICO

\title{
PARÁLISIS IDIOPÁTICA DEL NERVIO HIPOGLOSO. DESCRIPCIÓN DE UN CASO
}

\section{Idiopathic Hypoglossal Nerve Palsy. Case Report}

\author{
Yhovany GALLEGO-SÁNCHEZ'; Mikel VICENTE-PASCUAL ${ }^{1}$; Daniel VÁZQUEZ-JUSTES ${ }^{1}$; Leandro ANDREU- \\ MENCÍA ${ }^{2}$ \\ 1 Servicio Neurología. Hospital Universitari Arnau Vilanova, Lleida, España. \\ 2 Servicio de Otorrinolaringología. Hospital Universitari Arnau Vilanova, Lleida, España. \\ Correspondencia: mvpascual.1@gmail.com
}

Fecha de recepción: 22 de junio de 2020

Fecha de aceptación: 8 de agosto de 2020

Fecha de publicación: 16 de agosto de 2020

Fecha de publicación del fascículo: 1 de marzo de 2021

Conflicto de intereses: Los autores declaran no tener conflictos de intereses

Imágenes: Los autores declaran haber obtenido las imágenes con el permiso de los pacientes

Política de derechos y autoarchivo: se permite el autoarchivo de la versión post-print (SHERPA/RoMEO)

Licencia CC BY-NC-ND. Licencia Creative Commons Atribución-NoComercial-SinDerivar 4.0 Internacional

Universidad de Salamanca. Su comercialización está sujeta al permiso del editor

RESUMEN: Introducción: La parálisis del nervio hipogloso es una mononeuropatía poco frecuente. Las etiologías más habituales son la tumoral, la traumática, la isquémica cerebral y la iatrogénica. Descripción: Presentamos el caso de un paciente con neuropatía idiopática del nervio hipogloso, en el que, por edad, la presencia de factores de riesgo vascular, la microangiopatía cerebral y la evolución nos lleva a discutir acerca de la posibilidad de que se trate de una mononeuropatía isquémica o diabética como etiología del déficit. Discusión: En estudios publicados de parálisis idiopáticas del nervio hipogloso la edad de presentación se sitúa entre los 20 y 45 años y tienen una evolución y recuperación favorable, atribuyéndole un mecanismo fisiopatológico similar al de la parálisis de Bell. En nuestro caso la exploración física y las pruebas complementarias realizadas no mostraron etiología del déficit, encontrándonos ante un nuevo caso de parálisis idiopática del nervio hipogloso. Sin embargo, por la edad del paciente, la presencia de múltiples factores de riesgo vascular, la microangiopatía cerebral y la persistencia de la sintomatología nos hace plantear la 
PARÁLISIS IDIOPÁTICA DEL NERVIO HIPOGLOSO. DESCRIPCIÓN DE UN CASO

GALLEGO-SÁNCHEZ Y; VICENTE-PASCUAL M; VÁZQUEZ-JUSTES D; ANDREU-MENCÍA L

existencia de un mecanismo local, isquémico o diabético, como causa de la mononeuropatía presentada y pensar que nos podemos encontrar ante un mecanismo fisiopatológico similar al producido en otras mononeuropatías, como la del III o VI par craneal. Conclusión: Presentamos una parálisis del nervio hipogloso idiopática, que por las características de nuestro paciente nos hace pensar y discutir una posible etiología isquémica o diabética del déficit.

PALABRAS CLAVE: Nervio hipogloso; Parálisis; Mononeuropatía isquémica; Mononeuropatía diabética; Microangiopatía; Idiopático.

SUMMARY: Introduction: Hypoglossal nerve palsy is a rare mononeuropathy. The most common etiologies are tumor, traumatic, cerebral ischemic and iatrogenic causes. Description: We present a man with idiopathic hypoglossal nerve neuropathy. The patient's age, the presence of vascular risk factors and cerebral microangiopathy, and the clinical evolution leads us to discussion about the possibility of a ischemic or diabetic mononeuropathy as the etiology of the deficit. Discussion: In previous published series of idiopathic hypoglossal nerve palsy, the age of presentation is between 20 and 45 years and the patients have a favorable evolution. The pathophysiological mechanism could be similar to Bell's palsy. In our patient the physical examination and the complementary studies were normal, diagnose the deficit as idiopathic hypoglossal nerve palsy. However, due to patient's age, the presence of multiple vascular risk factors, cerebral microangiopathy and the persistence of the symptoms, we consider the existence of a local ischemic or diabetic damage as the cause of the mononeuropathy presented, and we think that the physiopathological mechanism could be similar to other mononeuropathies, such as III or VI cranial nerve palsy. Conclusion: We present an idiopathic hypoglossal nerve palsy, but due to characteristics of our patient, we discuss an ischemic or diabetic cause of the deficit.

KEYWORDS: Hypoglossal nerve; Paralysis; Ischemic mononeuropathy; Diabetic mononeuropathy; Microangiopathy; Idiopathic.

\section{INTRODUCCIÓN}

El nervio hipogloso es un nervio motor puro encargado de los movimientos de la lengua. Desde su salida a nivel del bulbo raquídeo abandona el cráneo a través del agujero condíleo anterior. Tras su salida del cráneo desciende uniéndose con ramas del plexo cervical, para posteriormente entrar en la cavidad oral, medial al ángulo mandibular [1]. Se han descrito múltiples etiologías de parálisis del nervio hipogloso, cobrando especial importancia en los últimos años la etiología idiopática. Presentamos el caso de una parálisis súbita del nervio hipogloso en un paciente con factores de riesgo vascular y microangiopatía, sin una causa etiológica demostrada, por lo que se podría considerar una parálisis idiopática. Sin embargo, por los antecedentes del paciente nos preguntamos y discutimos la posibilidad de una mononeuropatía isquémica diabética como causa del déficit.

\section{DESCRIPCIÓN}

Se trata de un varón de 83 años, exfumador, con antecedentes de diabetes mellitus tipo 2 en tratamiento con antidiabéticos orales, hipertensión arterial, dislipemia, síndrome de apnea hipopnea del sueño, infarto agudo de miocardio (con estenosis coronarias no revascularizadas) y miocardiopatía dilatada. Fue valorado en consultas externas de neurología por alteración del habla de inicio súbito. No refería disfagia, disfonía ni debilidad generalizada. A la exploración a las 3 semanas del inicio de la sintomatología se evidenció disartria moderada, paresia lingual izquierda y atrofia de hemilengua izquierda (Figura 1). El resto de la exploración 
neurológica fue anodina, con reflejos osteotendinosos presentes, salvo aquíleos, y sin fasciculaciones linguales ni musculares. Entre las exploraciones complementarias la analítica no mostró alteraciones relevantes, con serologías negativas. La RM cerebral descartó lesión isquémica a nivel bulbar, así como patología de fosa posterior, mostrando leucoaraiosis y lesiones de microangiopatía crónica. El estudio vascular mediante angioRM y dúplex de troncos supaaórticos y transcraneal no mostró disección carotidea. Una TC de cuello descartó patología infiltrativa local, con una exploración otorrinolaringológica que no mostró alteraciones en laringoscopia ni en la palpación de cuello. Dado lo súbito del cuadro y los factores de riesgo vascular entre sus antecedentes se orientó como mononeuropatía isquémica del XII par craneal izquierdo. Se mantuvo el tratamiento con antiagregación, y durante seis meses de seguimiento permanece la sintomatología, aunque ha mejorado la disartria inicial por la que consultó.

\section{DISCUSIÓN}

La etiología más frecuentemente descrita de paresia del nervio hipogloso es la etiología iatrogénica postquirúrgica, seguida de la tumoral y la idiopática [2]. Se han publicado casos más infrecuentes de etiología infecciosa [3-4], disecciones carotideas [5], granulomatosis de Wegener [6], enfermedad de Beçhet [7] y esclerosis lateral amiotrófica [8]. En los últimos años ha cobrado importancia la etiología idiopática, publicándose casos clínicos sin causa evidente tras estudios exhaustivos. En estos casos se supone un mecanismo similar a la parálisis facial idiopática o de Bell, siendo la edad de presentación más habitual en torno a los 20-45 años, con un pronóstico de recuperación favorable [9-11]. Como en dichas publicaciones, en nuestro paciente el estudio etiológico ha resultado negativo, encontrándonos ante un nuevo caso de parálisis de nervio hipogloso idiopático. Sin embargo, por la edad del paciente, la presencia de múltiples factores de riesgo vascular, la microangiopatía isquémica cerebral, el antecedente de infarto agudo de miocardio, junto con la persistencia de la sintomatología nos hace plantear la existencia de un mecanismo local, isquémico o diabético, como causa de la mononeuropatía presentada.

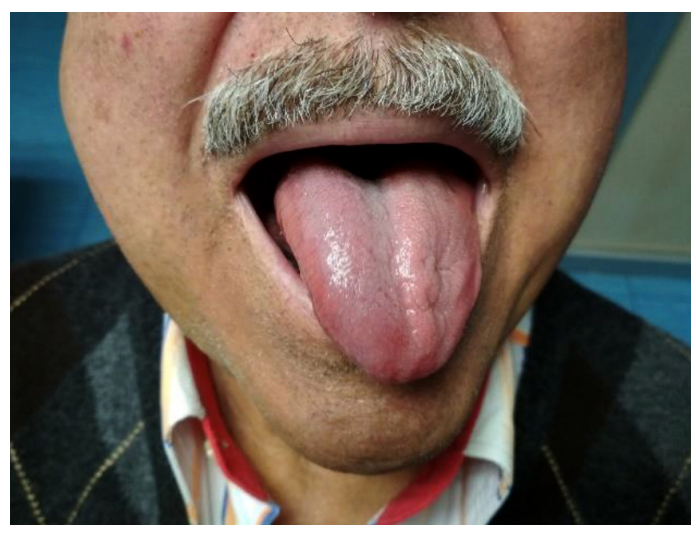

Figura 1. Desviación de la lengua hacia la izquierda. Atrofia de hemilengua izquierda.

En este caso el mecanismo fisiopatológico podría ser similar al producido en otras mononeuropatías, como la del III o VI par craneal. En la literatura hemos encontrado un caso similar, una paciente de 80 años en la cual la sintomatología persistió al cabo de 1 año, añadiéndose al tratamiento antiagregante plaquetario ante la sospecha de etiología embólica [12]. Dicha publicación también apoya que en casos de parálisis del nervio hipogloso etiquetados como idiopáticos, en presencia de factores de riesgo vascular, carga ateromatosa o microangiopatía cerebral, podría existir una etiología isquémica local de los vasa nervorum a lo largo del trayecto del nervio hipogloso y producirse una mononeuropatía isquémica del XII par craneal. Además en nuestro paciente no podría descartarse mononeuropatía diabética, tal como ocurre en otras neuropatías craneales. Pocos casos clínicos se han publicado al respecto [13], y en las series largas la etiología diabética es poco frecuente [2]. 
PARÁLISIS IDIOPÁTICA DEL NERVIO HIPOGLOSO. DESCRIPCIÓN DE UN CASO

GALLEGO-SÁNCHEZ Y; VICENTE-PASCUAL M; VÁZQUEZ-JUSTES D; ANDREU-MENCÍA L

\section{CONCLUSIONES}

Presentamos un nuevo caso de parálisis idiopática del nervio hipogloso, pero que por las características de nuestro paciente creemos que podría tratarse de una mononeuropatía isquémica o diabética, y que a su vez esta causa podría presentar un peor pronóstico de recuperación que en otras etiologías hasta ahora recogidas y abordadas en la literatura.

\section{AGRADECIMIENTOS}

El paciente ha dado su consentimiento oral y escrito para la publicación de su caso e imagen presentada. Desde aquí le queremos expresar nuestro más sincero agradecimiento, ya que este trabajo no hubiera sido posible sin su colaboración.

\section{BIBLIOGRAFÍA}

1. Carpenter M. Neuroanatomía: fundamentos. 4a ed. Buenos Aires: Editorial Médica Panamericana; 1999.

2. Stino AM, Smith BE, Temkit M, Reddy SN. Hypoglossal nerve palsy: 245 cases. Muscle Nerve. 2016;54:1050-4.

3. Parano E, Giuffrida S, Restivo D, Saponara R, Greco F, Trifiletti RR. Reversible palsy of the hypoglossal nerve complicating infectious mononucleosis in ayoung child. Neuropediatrics. 1998;29:46-7.

4. Johns MM, Hogikyan ND. Simultaneous vocal fold and tongue paresis secondary to Epstein- Barr virus infection. Arch Otolaryngol Head Neck Surg. 2000;126:1491-4.

5. Lindsay FW, Mullin D, Keefe MA. Subacute hypoglossal nerve paresis with internal carotid artery dissection. Laryngoscope. 2003;113:1530-3.

6. Finley JC Jr, Bloom DC, Thiringer JK. Wegener granulomatosis presenting as an infiltrative retropharyngeal mass with syncope and hypoglossal paresis. Arch Otolaryngol Head Neck Surg. 2004;130:361-5.

7. Ko KF, Kwan MC, Chan TP, Kay CS, Lam EK. Isolated hypoglossal nerve palsy in Behçet's disease. Ann Acad Med Singapore. 1997;26:848-9.

8. Goldstein ED, Eidelman BH. Misdiagnosis: Hypoglossal palsy mimicking bulbar onset amyotrophic lateral sclerosis. eNeurologicalSci. 2018;14:6-8.

9. Robaina Bordón JM, González Hernández A, Curutchet Mesner L, Gil Díaz A. Isolated hypoglossal nerve palsy. Neurologia. 2019;34:125-7.

10. Ahmed SV, Akram MS. Isolated unilateral idiopathic transienthypoglossal nervepalsy. BMJ Case Rep. 2014; 26;2014.

11. Shikino K, Noda K, Ikusaka M. Transient idiopathic isolated unilateral hypoglossal nerve palsy. J Gen Intern Med. 2013;28:591.

12. Nestola DF, Lombardi M, Brucoli M, Pia F, Aluffi P. Isolated Hypoglossal Nerve Palsy Mimicking a Base of Tongue Tumor. J Craniofac Surg. 2017;28:e78-e80.

13. Semiz S, Fişenk F, Akçurin S, Bircan I. Temporary multiple cranial nerve palsies in a patient with type 1 diabetes mellitus. Diabetes Metab. 2002;28:413-6. 